\title{
Clinical characteristics and gene mutation analysis of an adult patient with ETFDH-related multiple acyl-CoA dehydrogenase deficiency
}

\author{
CHENYI WANG* ${ }^{*}$ HAIHONG LV* ${ }^{*}$ XIA XU, YUPING MA and QIAN LI \\ Department of Endocrinology, The First Hospital of Lanzhou University, Lanzhou, Gansu 730000, P.R. China
}

Received March 8, 2020; Accepted August 27, 2020

DOI: $10.3892 / \mathrm{mmr} .2020 .11524$

\begin{abstract}
Multiple acyl-CoA dehydrogenase deficiency (MADD) is a rare autosomal recessive disorder of fatty acid metabolism caused by defects in electron transfer flavoprotein (ETF) or electron transfer flavoprotein dehydrogenase (ETFDH). These defects are mainly classified into the neonatal and late-onset types, based on their clinical manifestations. ETFDH gene mutations are generally considered to be associated with the late-onset type. The present study reported an adult woman with late-onset MADD accompanied with biochemical and muscle biopsy findings indicating metabolic disorders. Gene sequencing analysis showed that the c.1514T $>C$ homozygous mutation in the region of the 12th exon of the ETFDH gene, which led to the amino acid substitution p.I505T (isoleucine $>$ threonine), resulting in defective ETFDH protein function. The results of family verification revealed that the homozygous mutation originated from her parents. The female patient was treated with a large dose of vitamin B2, L-carnitine and coenzyme Q10, and the symptoms were significantly relieved. The c.1514T $>C$ mutation in the ETFDH gene, was considered as a novel pathogenic mutation that had not been previously reported. Therefore, it was hypothesized that this mutation was responsible for the clinical characteristics of the adult female patient. Overall, this novel mutation could expand the spectrum of the ETFDH gene mutation and provide the basis for the etiological and prenatal diagnosis of MADD.
\end{abstract}

Correspondence to: Professor Haihong Lv, Department of Endocrinology, The First Hospital of Lanzhou University, 1 Donggang West Road, Lanzhou, Gansu 730000, P.R. China

E-mail: haihonglv@126.com

*Contributed equally

Abbreviations: MADD, multiple acyl-CoA dehydrogenase deficiency; RR-MADD, riboflavin-responsive MADD; CoA, Coenzyme A; ETF, electron transfer flavoprotein; ETFDH, ETF dehydrogenase; FAD, flavin adenine dinucleotide

Key words: multiple acyl-CoA dehydrogenase deficiency, ETFDH, DNA sequencing, point mutation, riboflavin

\section{Introduction}

Multiple acyl-CoA dehydrogenase deficiency (MADD), a rare autosomal recessive disease, is a disorder of fatty acid oxidation caused by defects in electron transfer flavoprotein (ETF) or ETF dehydrogenase (ETFDH) (1). MADD can be divided into two subtypes, namely the neonatal and late-onset types, according to their clinical manifestations. The symptoms of late-onset MADD vary significantly, with muscle weakness being the most common symptom, while its most frequent pathological feature is lipid deposition in the muscle tissue. Late-onset MADD is the leading cause of lipid storage myopathy, and treatment with riboflavin has been considered to be effective (2-4). Several muscle diseases, such as inflammatory myopathies, metabolic myopathies and progressive muscular dystrophies, may also present with muscle weakness, therefore, MADD may be easily misdiagnosed as another type of muscle disease (5).

MADD was first described by Przyrembel et al (6) in 1976. The prevalence of MADD in the general population is estimated to be $\sim 9 / 1,000,000$ worldwide (7). Traditionally, muscle biopsy combined with biochemical tests to detect acylcarnitines and urine organic acids have been applied to diagnose MADD. However, gene sequencing can more precisely detect causative gene mutations. To date, published literature has reported $>160$ mutation sites in the ETFDH gene, but only a few repeat mutations (8). The present study reported the case of a patient who presented with muscle weakness and was diagnosed with MADD following genetic testing. Furthermore, the genetic analysis of the patient's parents identified a novel c.1514T $>C$ mutation located in the $4 \mathrm{Fe} 4 \mathrm{~S}$ domain of the ETFDH gene. Following timely and reasonable treatment, the patient's condition was significantly improved.

\section{Materials and methods}

Subjects and ethical approval. A 35-year-old female patient and her asymptomatic parents were enrolled at the First Hospital of Lanzhou University in February 2019. The present study was conducted with the approval of the Ethics Committee of the First Hospital of Lanzhou University (Lanzhou, China; approval no. LDYYLL2020-248). Written informed consent was obtained from the patient and her parents for participation in the study and publication of case details. The present 
study was performed in accordance with The Declaration of Helsinki.

Laboratory tests and muscle biopsy. Blood and urine samples were collected from the proband to determine the levels of acylcarnitines and organic acids, respectively. Urinary organic acids and blood acylcarnitines were analyzed by gas chromatography-mass spectrometry (GCMS-QP2010 analyzer; Shimadzu Corporation) and tandem mass spectrometry (ABI 2000; Applied Biosystems; Thermo Fisher Scientific, Inc.), respectively. For each examination, three scanning modes were used. For amino acids, the neutral loss scan was used (m/z 102) with the scanning range $\mathrm{m} / \mathrm{z} 140-280$. For acylcarnitines, the precursor scan of the product ion $\mathrm{m} / \mathrm{z} 85$ was used with the scanning range $\mathrm{m} / \mathrm{z} 210-502$. For glycine, ornithine, arginine and citrulline, multiple reaction monitoring (MRM) was used. GC-MS analysis was performed on an GCMS-QP2010 analyzer system. High-purity helium (99.9996\%) was used as the carrier gas at a constant flow rate of $1.2 \mathrm{ml} / \mathrm{min}$. The sample injection volume was $1 \mu \mathrm{l}$ and the split ratio was 10:1. The injection temperature was $300^{\circ} \mathrm{C}$ and the transfer line temperature was maintained at $280^{\circ} \mathrm{C}$. The column temperature was initially set at $70^{\circ} \mathrm{C}$ for $3 \mathrm{~min}$ and then increased to $300^{\circ} \mathrm{C}$ at $5^{\circ} \mathrm{C} / \mathrm{min}$ and held for $5 \mathrm{~min}$. The mass scan was set from 33 to 600 with a scan speed of 2 scans/sec. The diagnostic biopsy was performed on the left musculus biceps brachii. Briefly, following local infiltration anesthesia with $5 \mathrm{ml}$ of $2 \%$ lidocaine hydrochloride, an incision $\sim 2.5 \mathrm{~cm}$ in length was made at the maximum contraction of the muscle and a piece of muscle tissue $0.5 \times 0.8 \times 0.5 \mathrm{~cm}$ in size was harvested. Muscle specimens were frozen in isopentane that was precooled in liquid nitrogen and stored at $-80^{\circ} \mathrm{C}$. Furthermore, for histological examination, serial frozen sections $(8-\mu \mathrm{m}$ thick) were stained with hematoxylin and eosin, Oil Red O (ORO), modified Gomori trichrome and periodic acid Schiff. Briefly, cells were fixed with $10 \%$ formalin for $10 \mathrm{~min}$ at $25^{\circ} \mathrm{C}$ and treated with Oil Red O working solution for $5 \mathrm{~min}$ at $25^{\circ} \mathrm{C}$. Hematoxylin was added to the slide and incubated for $1 \mathrm{~min}$ at $25^{\circ} \mathrm{C}$, and then the slide was rinsed with tap water. Gomori staining solution was added for $20 \mathrm{~min}$ at $25^{\circ} \mathrm{C}$, followed by rinsing with tap water. Schiff's solution was stained for $10 \mathrm{~min}$ at $25^{\circ} \mathrm{C}$ and rinsed with water for $5 \mathrm{~min}$. Morphological alterations to muscle fibers were observed under a light microscope (magnification, $\mathrm{x} 40$ ).

Gene sequencing. Peripheral blood samples were collected from the proband and her parents in EDTA-treated tubes. The genomic DNA was extracted using the Blood Genome Column Medium Extraction kit (CWBio), according to the manufacturer's instructions. The extracted DNA was then subjected to the quality control process using a Qubit 2.0 fluorimeter and $0.8 \%$ agarose gel electrophoresis. Subsequently, protein-coding exome enrichment was performed using the xGen Exome Research Panel v1.0 (Integrated DNA Technologies, Inc.). This panel consists of 429,826 individually synthesized and quality-controlled probes that target $39 \mathrm{Mb}$ protein-coding regions (19,396 genes) of the human genome and cover $51 \mathrm{Mb}$ of end-to-end tiled probe space. Furthermore, high-throughput sequencing was carried out on a NovaSeq 6000 series sequencer (PE150; Illumina, Inc.), and $>99 \%$ of the target sequences were sequenced. The sequencing process was performed by Chigene Translational Medicine Research Center Co., Ltd. A search was conducted in the HGMDpro database for the novel mutation (https://digitalinsights.qiagen.com/products-overview/clinicalinsights-portfolio/human-gene-mutation-database/).

Bioinformatics analysis. Raw data were processed using fastp (version 0.18.1) (9) to remove adapter sequences and filter low quality reads. The Burrows-Wheeler Aligner (version 0.7.8) was used to align the paired-end reads to the Ensembl GRCh37/hg19 reference genome. Furthermore, the base quality score recalibration together with single nucleotide polymorphism (SNP) and short small insertions and deletion (indel) calling was performed using GATK (version 3.8) (10). SNPs and indels were screened to obtain high quality and reliable variants, depending on the sequencing depth and variant quality. In addition, an online system, independently developed by Chigene (www. chigene.org), was used to annotate database-based minor allele frequencies (MAFs), and the ACMG practice guideline-based pathogenicity of every yielded gene variant. The system also provided several software packages for the conservative analysis and protein product structure prediction. Several databases for MAF annotation were used, including 1000 genomes (www.1000genomes.org), dbSNP (http://www.ncbi. nlm.nih.gov/SNP), ESP (http://evs.gs.washington.edu/EVS/), ExAC (http://exac.broadinstitute.org/about), and the Chigene in-house MAF database. Additionally, for the prediction of the protein product structure variation, the following software packages were used: PROVEAN (version 1.1.3), SIFT (http://sift.jcvi.org/www/SIFT_seq_submit2.html), Polypen2_hdiv, Polypen2_hvar (http://genetics.bwh.harvard. edu/pph2/index.shtml), Mutationtaster2, M-Cap (version 1.4) and REVEL. To assess the pathogenicity of the genetic variation according to the ACMG guidelines, OMIM (http://www. ncbi.nlm.nih.gov/omim/limits), HGMD (http://www.hgmd. cf.ac.uk/ac/index.php) and ClinVar databases (https://www. ncbi.nlm.nih.gov/clinvar/) were used. Finally, to predict the functional changes of splice site variants, the MaxEntScan (http://genes.mit.edu/burgelab/maxent/Xmaxentscan_scoreseq. html), dbscSNV (11) and GTAG (www.chigene.org) software packages were applied, instead of the product structure prediction software. Computational mutation prediction of conserved domain analysis of the ETFDH gene was performed using NCBI/Structure/Cdd (https://www.ncbi.nlm.nih.gov/ Structure/cdd/wrpsb.cgi).

\section{Results}

Clinical characteristics and laboratory analysis. A 35-year-old woman was admitted to The First Hospital of Lanzhou University with progressively worsening limb weakness for $>2$ years. Since January 2016, the patient had been prone to fatigue without any obvious inducement. The female patient felt obvious weakness when she was going upstairs, which could be relieved after resting. The weakness in both lower limbs was aggravated in January 2017, and gradually she was not able to walk continuously, even for 20 meters. Gradually she exhibited weakness in both upper limbs and the nuchal region, and difficulty in raising her head, therefore, she was not capable for daily work. Subsequently, these symptoms 
Table I. Results of multiple muscle enzymes in this patient serum.

\begin{tabular}{|c|c|c|c|c|c|c|}
\hline Date & $\begin{array}{l}\text { AST (normal } \\
\text { range } 1-49 \mathrm{U} / 1 \text { ) }\end{array}$ & $\begin{array}{l}\text { ALT (normal } \\
\text { range } 1-49 \mathrm{U} / 1 \text { ) }\end{array}$ & $\begin{array}{c}\mathrm{LDH} \text { (normal } \\
\text { range } 125-240 \mathrm{U} / \mathrm{l})\end{array}$ & $\begin{array}{l}\alpha-\mathrm{HBDH} \text { (normal } \\
\text { range } 72-182 \mathrm{U} / \mathrm{l})\end{array}$ & $\begin{array}{c}\text { CK (normal } \\
\text { range 38-240 U/l) }\end{array}$ & $\begin{array}{l}\text { CK-MB (normal } \\
\text { range } 0-25 \mathrm{U} / \mathrm{l})\end{array}$ \\
\hline 28/07/2017 & 29 & / & 385 & 427 & 281 & 18 \\
\hline 07/08/2017 & 88 & 81 & 917 & 1,132 & 1,499 & 66 \\
\hline $11 / 08 / 2017$ & 32 & / & 591 & 658 & 442 & 23 \\
\hline $30 / 04 / 2018$ & 53 & I & 614 & 707 & 904 & 52 \\
\hline $19 / 06 / 2018$ & 47 & I & 556 & 635 & 551 & 36 \\
\hline $28 / 07 / 2018$ & 78 & 74 & 1,230 & 1,371 & 1,091 & I \\
\hline $15 / 08 / 2018$ & 35 & 41 & 178 & 96 & 107 & 20 \\
\hline
\end{tabular}

AST, aspartate transaminase; ALT, alanine transaminase; LDH, lactate dehydrogenase; $\alpha$-HBDH, $\alpha$-hydroxybutyrate; CK, creatine kinase; CK-MB, creatine kinase myocardial band.

were progressively aggravated. In July 2018, she was not able to speak and relied on a wheelchair for movement. During this period, she visited local hospitals several times, while the laboratory tests revealed elevated myozyme serum levels (Table I). However, she was misdiagnosed with polymyositis and progressive muscular dystrophy, and she underwent treatment with poor efficacy. She did not exhibit muscle twitching or muscle pain, choking cough when drinking water, or dyspnea. Her parents and siblings had no similar clinical symptoms. The pedigree of the proband is shown in Fig. 1. Neurological examination revealed that the muscle strength of the proximal limbs was grade IV, the distal muscle strength was grade IV, the neck muscle force was grade IV (Medical Research Council scale) (12), whereas the muscle tension was normal. The physiological reflexes were normal, while pathological ones were not elicited.

As shown in Table I, multiple muscle enzymes were elevated in the serum. Blood tandem mass spectrometry analysis revealed elevated $\mathrm{C} 4, \mathrm{C} 8$ and $\mathrm{C} 14: 1$ acylcarnitine levels, whereas, urine gas chromatography-mass spectrometry showed increased urinary excretion of 2-hydroxyglutaric acid, 3-hydroxyglutaric acid, 2-hydroxyisovaleric acid and ethylmalonic acid (Table II). Furthermore, blood glucose levels, electrolytes, infectious indicators, including C-reactive protein, procalcitonin and erythrocyte sedimentation rate, immunoglobulins, rheumatoid factors, autoantibody profiles, serum protein electrophoresis and endocrine hormone levels showed no significant abnormalities. Besides, a lower limb muscle magnetic resonance imaging scan indicated possible muscle inflammation. Additionally, electromyography showed myogenic damage, whereas abdominal ultrasound, echocardiography and craniocerebral computed tomography did not reveal any abnormalities (data not shown).

Muscle biopsy specimens exhibited a significant increase of the lipid droplets within the muscle fibers, which was consistent with the pathological characteristics of lipid storage myopathy. In addition, a few scattered atrophic myofibers were observed, 12-30 $\mu \mathrm{m}$ in diameter. Small vacuoles in uniform size were observed in several muscle fibers, while some vacuolar muscle fibers were slightly basophilic. Furthermore, ORO staining revealed a massive accumulation of lipid droplets within the vacuolar muscle fibers, with some of them fused

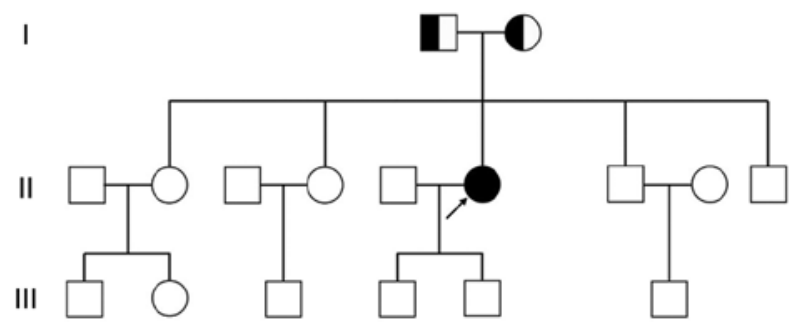

Figure 1. Pedigree of the proband. The proband (indicated by the arrow) carried c.1514T $>\mathrm{C}$ homozygous mutation, her parents were recessive carriers of the c.1514T $>\mathrm{C}$ mutation and showed no obvious symptoms, and this mutation was not found in any of her siblings.

into sheets. The aforementioned pathological changes may appear in MADD, therefore, its further diagnosis and classification needs to be determined by biochemical and genetic analyses (data not shown).

Genetic analysis. Whole exon sequencing revealed that the proband carried a c.4189A $>$ G (p.I1397V) heterozygous mutation and a c.1514T $>C$ (p.I505T) homozygous mutation, located in the dystrophin (DMD) and ETFDH genes, respectively (Fig. 2). No pathogenic mutations were detected in the ETFa and ETF $\beta$ genes.

The patient harbored a heterozygous mutation, c.4189A>G, in the 30th exon region of the DMD gene, resulting in the amino acid substitution p.I1397V (isoleucine $>$ valine), as shown in Fig. 2. For the female patient, the carrier status of the single heterozygous mutation should be classified as not pathogenic. Furthermore, DMD/Becker muscular dystrophy (BMD) gene mutations did not affect fatty acid metabolism and led to elevated serum levels of acylcarnitines and urine organic acids $(13,14)$, therefore, they were excluded.

The ETFDH gene exhibits autosomal recessive inheritance (1), therefore, pathogenic mutations on both alleles are likely to be pathogenic (homozygous or compound heterozygous mutations). A homozygous mutation, c.1514T $>C$, was identified in the 12th exon region of the ETFDH gene of the proband, leading to the amino acid substitution p.I505T (isoleucine $>$ threonine). The homozygous mutation originated from her parents (Fig. 2) and was consistent with an 
Table II. Blood acylcarnitine and urine organic acid test results of the proband.

\begin{tabular}{lccc}
\hline Item & Before treatment & After treatment & Reference range \\
\hline Blood acylcarnitine $(\mu \mathrm{mol} / \mathrm{l})$ & & & 0.39 \\
C4 & 2.10 & 0.08 & $0.17-0.91$ \\
C8 & 1.76 & 0.03 & $0.04-0.28$ \\
C14:1 & 0.43 & & 0.15 \\
Urinary organic acids (mmol/mol Cr) & & 6.70 & $<9.10$ \\
2-hydroxyglutaric acid & 14.70 & 0.10 & $<0.40$ \\
3-hydroxyglutaric acid & 2.18 & 1.25 & $<2.26$ \\
2-hydroxyisovaleric acid & 3.90 & 0.48 & $<4.14$ \\
Ethylmalonic acid & 7.62 & & \\
\hline
\end{tabular}

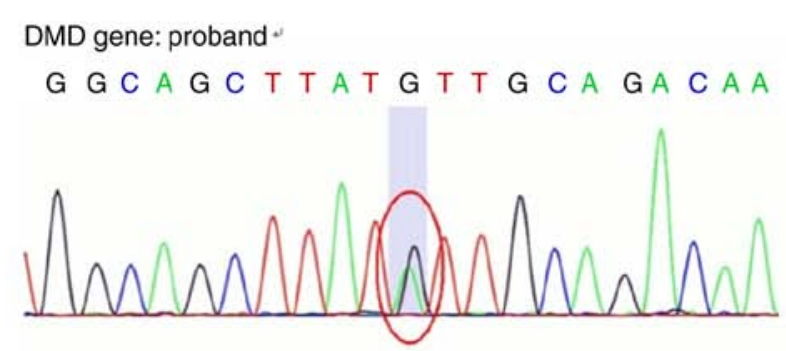

DMD gene: proband's father.

T T G T C T G CAATA TA G T T C C

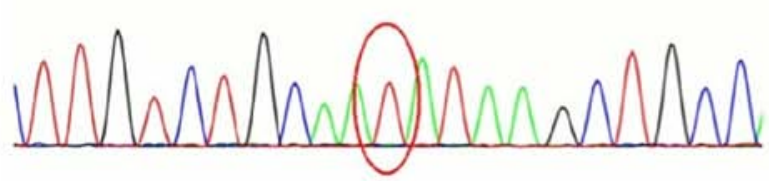

DMD gene: proband's mother

T T G T C T G CA A A T A A G C T G C

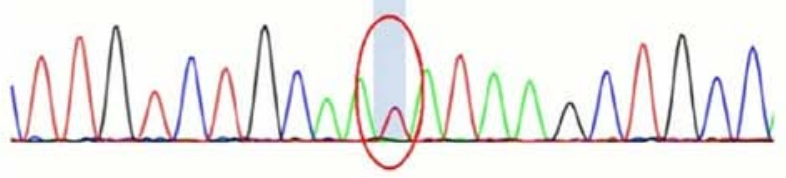

ETFDH gene: proband

$T G C A C A C C T A C T G A G T A T C C A$

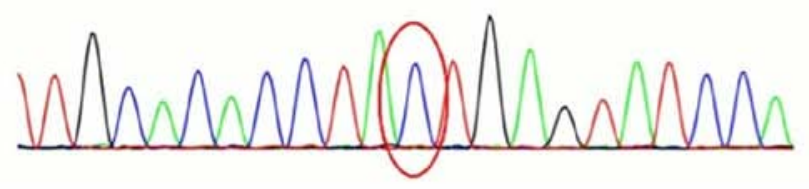

ETFDH gene: proband's father

$T G C A C A C C T A T T G A G T A T C C A$

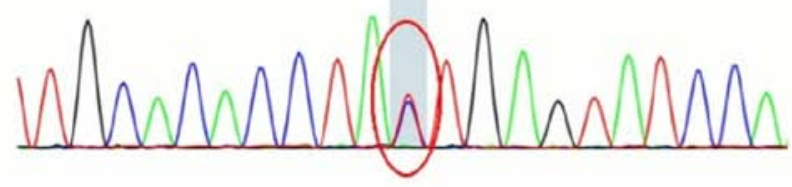

ETFDH gene: proband's mother *

$T G C A C A C C T A T T G A G T A T C C A$

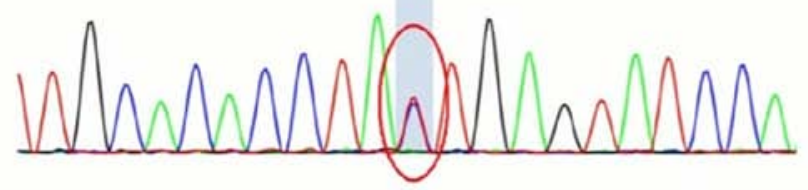

Figure 2. Sanger sequencing of the patient and her parents. The proband carried c.4189A $>$ G heterozygous mutation of the DMD gene, her father had no mutation of this gene, and her mother carried c.4189A $>\mathrm{G}$ heterozygous mutation of this gene. The proband carried c.1514T $>\mathrm{C}$ homozygous mutation of the ETFDH gene, and both of her parents carried the c.1514T $>$ C heterozygous mutation. DMD, dystrophin; ETFDH, electron transfer flavoprotein dehydrogenase.

autosomal recessive mode of inheritance. Mutations in the ETFDH gene are often associated with impaired fatty acid oxidation (3), which is consistent with the laboratory findings of this patient.

Computational mutation prediction of conserved domain analysis of the ETFDH gene was performed using the $\mathrm{NCBI} /$ Structure/Cdd. The analysis revealed a mutation, resulting in the substitution of the 505th amino acid from isoleucine to threonine, located in the $4 \mathrm{Fe}-4 \mathrm{~S}$ domain of the ETFDH gene (Fig. 3). ETFDH in the inner mitochondrial membrane accepts electrons from ETF, located in the mitochondrial matrix, and reduces ubiquinone in the mitochondrial membrane $(7,8)$. The two redox centers of the protein, flavin adenine dinucleotide (FAD) and the $4 \mathrm{Fe}-4 \mathrm{~S}$ cluster, are present in a $64-\mathrm{kDa}$ monomer structure domain. According to the NCBI/Structure/Cdd, a sequence-based mutation prediction for p.I505T, indicated that this amino acid substitution could affect the function of ETFDH.

Diagnosis and treatment. Based on the mutation analysis by gene sequencing, combined with the clinical manifestations and laboratory findings, the patient was diagnosed with 

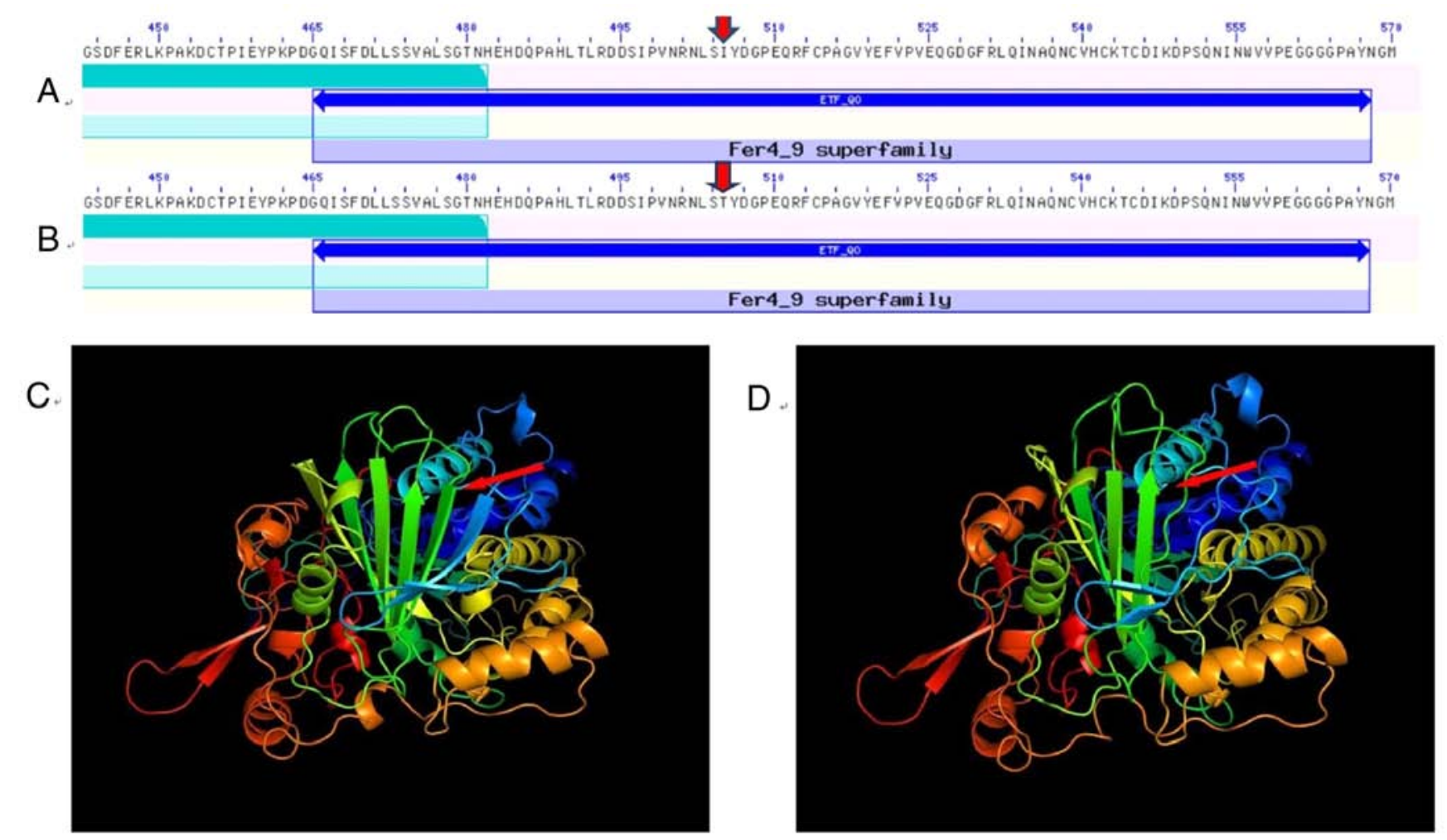

Figure 3. Computational mutation prediction. (A) Protein sequence of wild-type ETFDH. The site indicated by the red arrow is the isoleucine at position 505, which is located within the $4 \mathrm{Fe} 4 \mathrm{~S}$ cluster domain. (B) The protein sequence of the proband. The site indicated by the red arrow is the threonine at position 505 . (C) The three-dimensional structure of wild-type ETFDH and (D) the three-dimensional structure of the ETFDH from the proband. ETFDH, electron transfer flavoprotein dehydrogenase.

MADD. Therefore, she was immediately treated with $80 \mathrm{mg}$ vitamin B2 three times daily, $1 \mathrm{~g}$ L-carnitine twice a day and $50 \mathrm{mg}$ coenzyme Q10 three times daily, and her health status was significantly improved (Tables I and II).

\section{Discussion}

MADD, also known as glutaric acidemia type II, is characterized by elevated urine organic acid levels and is clinically divided into three subtypes, namely the neonatal-onset with congenital malformations MADD (type I), the neonatal-onset without congenital malformations MADD (type II), and mild or late-onset MADD (type III) $(3,15,16)$. The first two subtypes are often characterized by severe multiple acyl-CoA dehydrogenation defects. Due to the rapid onset and severe phenotype, the majority of children die shortly after disease onset. Unlikely, the clinical phenotypes and age at presentation of late-onset MADD are highly variable, ranging from early infancy to late adulthood. This type of MADD occurs insidiously, and the majority of patients present with intermittent muscle weakness, paroxysmal vomiting, hypoglycemia and other clinical features, including sensory neuropathy, tendon reflex disappearance and fatty liver disease $(15,17)$. Due to the lack of specific clinical manifestations, late-onset MADD may easily be misdiagnosed as myositis, progressive muscular dystrophy and glycogen storage disease (5).

MSDD is caused by ETF or ETFDH deficiencies. ETF is a heterodimer located in the mitochondrial matrix, consisting of two subunits, namely $\alpha$ and $\beta$, which are encoded by the
ETF $\alpha$ and ETF $\beta$ gene, respectively. Furthermore, the ETFDH protein is located in the inner mitochondrial membrane and encoded by the ETFDH gene. ETFDH accepts electrons from ETF and transmits them to ubiquinone. Its crystal structure is comprised of three functional domains: The FAD, $4 \mathrm{Fe}-4 \mathrm{~S}$ cluster and coenzyme Q10 binding domains (Fig. 3). The genes encoding ETF $\alpha$, ETF $\beta$ and ETFDH are located at 15p23-25, $19 q 13$ and 4q33, respectively $(18,19)$. ETF and ETFDH link the oxidation of fatty acids and some amino acids to the mitochondrial oxidative respiratory chain, therefore, both proteins serve an important role in the process of electron transfer (8). Mutations in any of the ETF $\alpha$, ETF $\beta$ and ETFDH genes may lead to functional defects of the corresponding proteins, thus resulting in metabolism disorders of the fatty acids and other substances, insufficient energy production and muscle weakness accompanied by lipid deposition in muscle tissues (20).

In the past, the diagnosis of MADD could be quickly and easily achieved using tandem mass spectrometry of blood samples, which could be further confirmed by gas chromatography mass spectrometry analysis of urine organic acids $(21,22)$. However, in late-onset patients, the increased levels of acylcarnitines and urinary organic acids may be mild and atypical, or detectable only during acute episodes $(5,17)$, therefore, genetic analysis to detect mutations in the ETF or ETFDH genes is considered as the key method for a definitive diagnosis.

The conserved domain analysis of the ETFDH gene revealed that the $c .1514 \mathrm{~T}>\mathrm{C}$ mutation, resulting in the substitution of isoleucine with threonine, was located 
in the $4 \mathrm{Fe}-4 \mathrm{~S}$ domain of the ETFDH gene. Wen et al (3) and Wang et al (23) demonstrated that the vast majority of ETFDH mutations were concentrated in the FAD-binding domain, followed by the coenzyme Q10 binding domain. However, mutations within the $4 \mathrm{Fe}-4 \mathrm{~S}$ cluster structural domain were rare. Although the $4 \mathrm{Fe}-4 \mathrm{~S}$ cluster binding domain does not directly bind and transfer electrons, it is involved in electron transfer by acting as a cofactor. Usselman et al (24) revealed that amino acid variations in the $4 \mathrm{Fe}-4 \mathrm{~S}$ cluster could attenuate the activity of ubiquinone reductase and slow down the electron transfer rate to the oxidative respiratory chain. A search in the HGMDpro database and the retrieval of several major public databases revealed that the $\mathrm{c} .1514 \mathrm{~T}>\mathrm{C}$ mutation had not been previously reported, suggesting that this mutation was extremely rare in the general population. The novel mutation site was located in the $4 \mathrm{Fe}-4 \mathrm{~S}$ cluster domain, which combined with the previously reported mutations, broadened the ETFDH gene mutation spectrum.

Previous studies have reported that some patients with MADD are sensitive to riboflavin, also known as riboflavin-responsive MADD (RR-MADD), which is more common in the late-onset type $(3,5,25)$. However, there is no specific treatment for RR-MADD, and patients are usually treated with riboflavin accompanied with L-carnitine and coenzyme Q10 administration as adjuvant therapy. In this case, when treatment is accompanied by a low fat, low protein and high carbohydrate diet, the effects are significant for the patient's health. Therefore, the symptoms of muscle weakness are significantly improved, and the abnormal laboratory parameters are also gradually corrected. However, the exact mechanism underlying the effect of riboflavin in the treatment of MADD remains unclear. It has been suggested that FAD may act as a molecular chaperone that mediates the proper folding of certain flavoproteins. Therefore, the elevated FAD concentration within mitochondria may compensate for the decreased folding capacity of the mutated proteins. As a precursor of the coenzyme FAD, riboflavin therapy may enhance the stability of mutant flavoproteins and the activity of the ETFDH proteins $(23,25)$. As there are only a few previous reports regarding mutations in the $4 \mathrm{Fe}-4 \mathrm{~S}$ domain, it is therefore difficult to determine the pathophysiological mechanisms of riboflavin treatment on such mutations. We speculate that mutations in the $4 \mathrm{Fe}-4 \mathrm{~S}$ domain may not only lead to reduced structural stability of the ETFDH protein, but may also affect the activity of ubiquitin reductase. Therefore, increasing FAD concentration may partially compensate for these defects. Besides, it has been demonstrated that in recessive genetic diseases, the phenotype of the patient is often determined by the milder genetic variant when different mutations are present in the two alleles (25). The present study indicated that the c.1514T $>$ C mutation was a mild ETFDH gene mutation, which could also partially explain why this patient was very sensitive to riboflavin treatment.

The association between the genotype and clinical phenotype in patients with MADD has been extensively studied. Although some patients carry the same mutation, the onset time and clinical manifestations vary among them (5), indicating that the environment may have an impact on the phenotype. However, further studies are still needed to elucidate these differences. In the present study the patient suffered from late-onset MADD and only showed intermittent muscle weakness, and no other clinical manifestations such as myalgia, hypoglycemia or severe fatty liver disease, which further illustrated the high heterogeneity of the disease.

In conclusion, a case of late-onset MADD with limb weakness and exercise intolerance as the first symptoms was reported. Genetic analysis revealed that the c.1514T $>C$ was a pathogenic mutation, which broadened the spectrum of the ETFDH gene mutations and provided a valuable reference for early diagnosis and treatment. Special consideration should be given to adult patients with unexplained myasthenia and elevated muscle enzyme levels to the possibility of late-onset MADD. When muscle biopsy indicates lipid storage myopathy, genetic testing should be performed to further confirm the first diagnosis. Nevertheless, early diagnosis and reasonable treatment may effectively improve the prognosis of patients with MADD.

\section{Acknowledgements}

Not applicable.

\section{Funding}

No funding was received.

\section{Availability of data and materials}

The datasets used and/or analyzed during the current study are available from the corresponding author on reasonable request.

\section{Authors' contributions}

HL conceived the present study, participated in its methodology design, drafted the manuscript and interpreted the data. CW conceptualized the study, obtained molecular genetic literature, drafted the manuscript and interpreted data. CW and YM acquired the data. Data analysis was performed by XX and YM. YM obtained molecular genetic literature and coordinated the study. Resources and interpretation of data were provided and performed by CW, XX and QL. All authors read and approved the final manuscript.

\section{Ethics approval and consent to participate}

This study was conducted with approval from the Ethics Committee of The First Hospital of Lanzhou University and in accordance with the Declaration of Helsinki. Written informed consent was obtained from the patient and her parents for participation in the study.

\section{Patient consent for publication}

Written informed consent was obtained from the patient and her parents for publication of case details. 


\section{Competing interests}

The authors declare that they have no competing interests.

\section{References}

1. Frerman FE and Goodman SI: Deficiency of electron transfer flavoprotein or electron transfer flavoprotein: Ubiquinone oxidoreductase in glutaric acidemia type II fibroblasts. Proc Natl Acad Sci USA 82: 4517-4520, 1985.

2. Vergani L, Barile M, Angelini C, Burlina AB, Nijtmans L, Freda MP, Brizio C, Zerbetto E and Dabbeni-Sala F: Riboflavin therapy. Biochemical heterogeneity in two adult lipid storage myopathies. Brain 122: 2401-2411, 1999.

3. Wen B, Dai T, Li W, Zhao Y, Liu S, Zhang C, Li H, Wu J, Li D and Yan C: Riboflavin-responsive lipid-storage myopathy caused by ETFDH gene mutations. J Neurol Neurosurg Psychiatry 81 : 231-236, 2010.

4. Nilipour Y, Fatehi F, Sanatinia S, Bradshaw A, Duff J, Lochmüller H, Horvath R and Nafissi S: Multiple acyl-coenzyme A dehydrogenase deficiency shows a possible founder effect and is the most frequent cause of lipid storage myopathy in Iran. J Neurol Sci 411: 116707, 2020.

5. Fu HX, Liu XY, Wang ZQ, Jin M, Wang DN, He JJ, Lin MT and Wang N: Significant clinical heterogeneity with similar ETFDH genotype in three Chinese patients with late-onset multiple acyl-CoA dehydrogenase deficiency. Neurol Sci 37: 1099-1105, 2016.

6. Przyrembel H, Wendel U, Becker K, Bremer HJ, Bruinvis L, Ketting D and Wadman SK: Glutaric aciduria type II: Report on a previously undescribed metabolic disorder. Clin Chim Acta 66: 227-239, 1976.

7. Chautard R, Laroche-Raynaud C, Lia AS, Chazelas P, Derouault P, Sturtz F, Baaj Y, Veauville-Merllié A, Acquaviva C, Favreau F and Faye PA: A case report of a mild form of multiple acyl-CoA dehydrogenase deficiency due to compound heterozygous mutations in the ETFA gene. BMC Med Genom 13: 12, 2020.

8. Lucas TG, Henriques BJ and Gomes CM: Conformational analysis of the riboflavin-responsive ETF:QO-p.Pro456Leu variant associated with mild multiple acyl-CoA dehydrogenase deficiency. Biochim Biophys Acta Proteins Proteom 1868: 140393, 2020.

9. Chen S, Zhou Y, Chen Y and Gu J: fastp: An ultra-fast all-in-one FASTQ preprocessor. Bioinformatics 34: i884-i890, 2018.

10. McKenna A, Hanna M, Banks E, Sivachenko A, Cibulskis K, Kernytsky A, Garimella K, Altshuler D, Gabriel S, Daly M and DePristo MA: The Genome Analysis Toolkit: A MapReduce framework for analyzing next-generation DNA sequencing data Genome Res 20: 1297-1303, 2010.

11. Jian X, Boerwinkle E and Liu X: In silico prediction of splice-altering single nucleotide variants in the human genome. Nucleic Acids Res 42: 13534-13544, 2014.
12. Paternostro-Sluga T, Grim-Stieger M, Posch M, Schuhfried O, Vacariu G, Mittermaier C, Bittner C and Fialka-Moser V: Reliability and validity of the Medical Research Council (MRC) scale and a modified scale for testing muscle strength in patients with radial palsy. J Rehabil Med 40: 665-671, 2008.

13. Koenig M, Beggs AH, Moyer M, Scherpf S, Heindrich K, Bettecken T, Meng G, Müller CR, Lindlöf M, Kaariainen H, et al: The molecular basis for Duchenne versus Becker Muscular Dystrophy: Correlation of severity with type of deletion. Am J Hum Genet 45: 498-506, 1989.

14. Moser H: Duchenne muscular dystrophy: Pathogenetic aspects and genetic prevention. Human Genetics 66: 17-40, 1984.

15. Goodman SI and Frerman FE: Glutaric acidaemia type II (multiple Acyl-Coa dehydrogenation deficiency). J Inherit Metab Dis 7: $33-37,1984$.

16. Gordon N: Glutaric aciduria types I and II. Brain Dev 28: 136-140, 2005.

17. al-Essa MA, Rashed MS, Bakheet SM, Patay ZJ and Ozand PT: Glutaric aciduria type II: Observations in seven patients with neonatal- and late-onset disease. J Perinatol 20: 120-128, 2000.

18. Schiff M,Froissart R, Olsen RK, Acquaviva C and Vianey-Saban C: Electron transfer flavoprotein deficiency: Functional and molecular aspects. Mol Genet Metab 88: 153-158, 2006.

19. Zhang J, Frerman FE and Kim JJ: Structure of electron transfer flavoprotein-ubiquinone oxidoreductase and electron transfer to the mitochondrial ubiquinone pool. Proc Natl Acad Sci USA 103: 16212-16217, 2006.

20. Angelini C, Tavian D and Missaglia S: Heterogeneous phenotypes in lipid storage myopathy Due to ETFDH gene mutations. JIMD Rep 38: 33-40, 2018.

21. Champion M: An approach to the diagnosis of inherited metabolic disease. Arch Dis Child Educ Pract Ed 95: 40-46, 2010

22. Li Q, Wei S, Wu D, Wen C and Zhou J: Urinary metabolomics study of patients with gout using gas chromatography-mass spectrometry. Biomed Res Int 2018: 3461572, 2018.

23. Wang ZQ, Chen XJ, Murong SX, Wang N and Wu ZY: Molecular analysis of 51 unrelated pedigrees with late-onset multiple acyl-CoA dehydrogenation deficiency (MADD) in southern China confirmed the most common ETFDH mutation and high carrier frequency of c. 250G $>$ A. J Mol Med (Berl) 89: 569-576, 2011.

24. Usselman RJ, Fielding AJ, Frerman FE, Watmough NJ, Eaton GR and Eaton SS: Impact of mutations on the midpoint potential of the $[4 \mathrm{Fe}-4 \mathrm{~S}]+1,+2$ cluster and on catalytic activity in electron transfer flavoprotein-ubiquinone oxidoreductase (ETF-QO). Biochemistry 47: 92-100, 2008.

25. Olsen RKJ, Olpin SE, Andresen BS, Miedzybrodzka ZH, Pourfarzam M, Merinero B, Frerman FE, Beresford MW, Dean JC, Cornelius N, et al: ETFDH mutations as a major cause of riboflavin-responsive multiple acyl-CoA dehydrogenation deficiency. Brain 130: 2045-2054, 2007.

This work is licensed under a Creative Commons Attribution-NonCommercial-NoDerivatives 4.0 International (CC BY-NC-ND 4.0) License. 